\title{
Temporal cavity solitons
}

\author{
Time-domain cavity solitons could be a promising candidate for creating all-optical buffers integrated \\ with pulse reshapers and wavelength converters. Stéphane Coen spoke with Nature Photonics about the \\ generation of these solitons and their potential applications in telecommunications.
}

Tell us about your work.

Our work experimentally demonstrates a special class of solitons that we call 'temporal cavity solitons' in an externally driven passive nonlinear Fabry-Pérot resonator. The findings show that short light pulses of around four picoseconds can be held in a passive fibre loop for more than a second - a time that is limited only by environmental perturbations. We have also used solitons to store binary data and have studied the interactions between adjacent solitons. Our findings reveal that these solitons can be packed at high density and that an all-optical buffer based on this technology is viable, in principle.

What is the physical mechanism behind your work?

The mechanism that leads to the existence of these temporal cavity solitons results from a combination of the chromatic dispersion and Kerr nonlinearity of silica fibres (that is, their intensity-dependent refractive index), together with the feedback and dissipation provided by a Fabry-Pérot cavity geometry. Chromatic dispersion, which normally broadens such pulses, is compensated by the Kerr nonlinearity of the fibre, making these solitons much like classic solitons in that their shape is maintained. The distinct feature of our work is that the external driving beam compensates for the energy lost every time the solitons complete a roundtrip of the fibre loop. Although this external driving beam is just a simple continuouswave beam, it is able to maintain the pulses undistorted, roundtrip after roundtrip, for as long as it is present. If the driving or holding beam is turned off, the solitons dissipate all of their energy over a few roundtrips. These cavity solitons are therefore a subclass of the more widely known dissipative solitons that exist, for example, in fibre lasers. This mechanism may sound complicated, but in reality it is not; we are dealing with a simple closed fibre loop, and this simplicity gives an interesting fundamental twist to our research. Many systems in nature exhibit nonlinearity, dissipation and a mechanism similar to diffusion that connects different parts of an extended system. Essentially this combination can be found in all

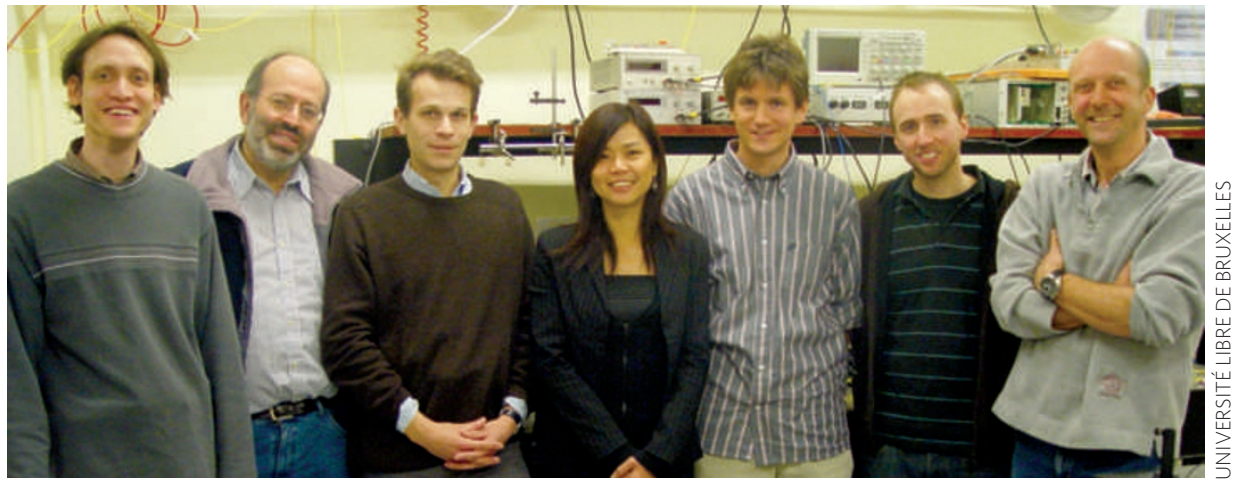

From left to right: Simon-Pierre Gorza, Philippe Emplit, Pascal Kockaert, Rachel Won (Nature Photonics), Stéphane Coen, François Leo and Marc Haelterman.

living organisms, and it is what gives rise to all the natural patterns one can find in nature, such as animal spots and so on. This is related to the concept of dissipative structures introduced by Nobel Prize winner Ilya Prigogine. Because our study has some connection to that work, we believe that it may well be the most fundamental example of self-organization in nonlinear optics.

\section{What are the potential applications?}

We have demonstrated that several temporal cavity solitons can be stored in a cavity, one after the other, and that they keep circulating around the loop. This immediately suggests that these solitons could be used for all-optical memory or buffering applications. The advantage of such an all-optical buffering scheme is that the intrinsic properties of the cavity solitons would confer some very promising features. For example, cavity solitons can be excited or written inside the fibre loop by sending a short pulse at a different wavelength (the writing pulse) that interacts with the driving beam through cross-phase modulation. This automatically provides wavelength conversion at the cavity input as the stored cavity solitons have a different wavelength to that of the writing pulses. Furthermore, cavity solitons form an attracting state in which the writing pulse can have a temporal shape or power level not quite matching those of the cavity solitons, yet the pulse ultimately stored in the cavity will always be reshaped into the unique profile of the cavity solitons. This naturally offers an all-optical reshaping functionality that is critical for telecommunications applications. All of these features show that temporal cavity solitons are probably more naturally compatible with signal processing and telecommunications applications than their spatial counterparts.

\section{What are the challenges and future work?}

The main challenges are to improve the interferometric stability of the cavity, and ideally to design a scheme that can selfstabilize. Also, it currently takes around 100 roundtrips to stabilize the solitons. This can be sped up by using a shorter cavity length and a higher input power. Fibres with a higher nonlinearity or on-chip microresonators can then be used to reduce the detrimental high input power. Note that the cavity length can't be too short as this would leave no space for data storage. In the future, apart from improving the stabilization of the cavity length, we would like to experimentally demonstrate the optical erasure of temporal cavity solitons and also look further into the interactions between paired solitons.

\section{INTERVIEW BY RACHEL WON}

Stéphane Coen and his co-workers have an Article on their temporal cavity solitons on page 471 of this issue. 